\title{
Supporting Information for Data-Driven Robust Optimization Based on Principle Component Analysis and Cutting Plane Methods
}

\author{
Shulei Zhang ${ }^{\mathrm{a}}$, Runda Jia ${ }^{\mathrm{a}, \mathrm{b}, \mathrm{c}, *}$, Dakuo He ${ }^{\mathrm{a}, \mathrm{b}}$, Fei Chu ${ }^{\mathrm{d}}$ \\ a School of Information Science \& Engineering, Northeastern University, Shenyang 110004, \\ China \\ b State Key Laboratory of Synthetical Automation for Process Industries, Northeastern University, \\ Shenyang 110004, China \\ ${ }^{\mathrm{c}}$ Liaoning Key Laboratory of Intelligent Diagnosis and Safety for Metallurgical Industry, \\ Northeastern University, Shenyang 110004, China \\ d School of Information and Control Engineering, China University of Mining and Technology, \\ Xuzhou 221116, China
}

\section{Contents}

Appendix A: Details of principle component analysis .2

Appendix B: Supplementary data for scheduling application .3

Appendix C: Dual formulations for second-stage of the ARO model for scheduling application .4

\footnotetext{
${ }^{*}$ Corresponding author. Tel.: +86 24 83672320; fax: +86 2483672320.

E-mail address: jiarunda@ise.neu.edu.cn (R.D. Jia).
} 



\section{Appendix A: Details of principal component analysis}

In this appendix, the details of PCA are given.

The optimization objective of PCA is to maximize the sum of the variances of the data mapped to the PCs. At first, we define the PCs as $\mathbf{r}_{i} \in \mathrm{R}^{m}$, where $i=1, \mathrm{~K}, m$. Besides, we set $\mathbf{r}_{i}^{\mathrm{T}} \mathbf{r}_{i}=1$ and $\mathbf{r}_{i}^{\mathrm{T}} \mathbf{r}_{j}=0$, where $j=1, \mathrm{~K}, m, j \neq i$.

Since $\because$ is the scaled matrix of the uncertainty data matrix $\mathbf{U}$, the sum of the variances of the scaled uncertainty data mapped to the $\mathrm{PC}_{i}$ can be formulated as

$$
V S=\mathbf{r}_{i}^{\mathrm{T}} \mathbf{U}^{\mathrm{d}} \mathbf{U}^{\mathrm{U}} \mathbf{r}_{i}
$$

We set $i=1$, and the optimization objective can be formulated as shown in Eq. (A.2).

$$
\begin{aligned}
& V S=\max _{\mathbf{r}_{\mathbf{1}}} \frac{1}{N-1} \mathbf{r}_{1}^{\mathrm{T}} \mathbf{U}^{\delta} \mathbf{U}_{\mathbf{r}_{1}} \\
& \text { s.t } \mathbf{r}_{1}^{\mathrm{T}} \mathbf{r}_{1}=1
\end{aligned}
$$

It should be noted the item $1 / N-1$ added into the objective function does not affect the optimization solutions. Therefore, Eq. (A.2) can be further reformulated as follows.

$$
\begin{aligned}
& V S=\max _{\mathbf{r}_{1}} \mathbf{r}_{1}^{\mathrm{T}} \mathbf{S} \mathbf{r}_{1} \\
& \text { s.t } \mathbf{r}_{1}^{\mathrm{T}} \mathbf{r}_{1}=1
\end{aligned}
$$

By introducing the Lagrange multiplier $\mu$, the optimization problem (A.3) can be constructed as follows.

$$
V S=\max _{\mathbf{r}_{1}} \mathbf{r}_{1}^{\mathrm{T}} \mathbf{S r}_{1}+\mu\left(1-\mathbf{r}_{1}^{\mathrm{T}} \mathbf{r}_{1}\right)
$$

Taking the derivative of the Eq. (A.4) with respect to $\mathbf{r}_{1}$ and equating that to zero, we can obtain the following equation.

$$
\mathbf{S r}_{1}=\mu \mathbf{r}_{1}
$$


If both sides of the Eq. (A.5) are left multiplied by $\mathbf{r}_{1}^{\mathrm{T}}, \mathbf{r}_{1}^{\mathrm{T}} \mathbf{S} \mathbf{r}_{1}=\mu$ can be obtained. Therefore, the objective value is maximized when $\mu$ is the largest eigenvalue of the matrix $\mathbf{S}$ and $\mathbf{r}_{1}$ is the corresponding eigenvector. Combined with the introduction in section 2.2, we can obtain $\mathbf{r}_{1}=\mathbf{p}_{1}$.

The remaining $\mathbf{r}_{i}$ can be calculated by the following optimization problem.

$$
\begin{aligned}
& V S_{i}=\max _{\mathbf{r}_{i}} \mathbf{r}_{i}^{\mathrm{T}} \mathbf{S r}_{i} \\
& \text { s.t } \mathbf{r}_{i}^{\mathrm{T}} \mathbf{r}_{i}=1 \\
& \mathbf{r}_{i}^{\mathrm{T}} \mathbf{r}_{j}=0 \\
& i=2, \mathrm{~K}, m \\
& j=1, \mathrm{~K}, m \text { and } j \neq i
\end{aligned}
$$

Similarly, we can obtain that $\mathbf{r}_{i}=\mathbf{p}_{i}$. Therefore, the new coordinate system or PCs is equal to the matrix $\mathbf{P}$.

\section{Appendix B: Supplementary data for scheduling application}

Table S1. Data for materials in the scheduling application

\begin{tabular}{ccccccccccccccc}
\hline States & M1 & M2 & M3 & M4 & I1 & I2 & I3 & I4 & I5 & I6 & P1 & P2 & P3 & P4 \\
\hline Capacity(kg) & 1000 & 1000 & 1000 & 1000 & 1000 & 1000 & 1000 & 1000 & 1000 & 1000 & 1000 & 1000 & 1000 & 1000 \\
Initial load & 1000 & 1000 & 1000 & 1000 & 0 & 0 & 0 & 0 & 0 & 0 & 0 & 0 & 0 & 0 \\
$\quad(\mathrm{Kg})$ & & & & & & & & & & & & & & \\
Price $(\$ / K g)$ & 0 & 0 & 0 & 0 & 0 & 0 & 0 & 0 & 0 & 0 & 39.78 & 30.52 & 15.08 & 5.14 \\
Demand & 0 & 0 & 0 & 0 & 0 & 0 & 0 & 0 & 0 & 0 & 138.1 & 178.4 & 120.8 & 101.7 \\
$(\mathrm{Kg})$ & & & & & & & & & & & & & & \\
\hline
\end{tabular}

Table S2. Data for tasks in the scheduling application

\begin{tabular}{ccccccc}
\hline Tasks & $\begin{array}{c}\text { Fixed cost } \\
(\$)\end{array}$ & $\begin{array}{c}\text { Variable cost } \\
(\$ / \mathrm{Kg})\end{array}$ & $\begin{array}{c}\text { Fixed } \\
\text { processing } \\
\text { Time }(\mathrm{h})\end{array}$ & $\begin{array}{c}\text { Variable } \\
\text { processing } \\
\text { Time }(\mathrm{h})\end{array}$ & $\begin{array}{c}\text { Minimum } \\
\text { batch size } \\
(\mathrm{Kg})\end{array}$ & $\begin{array}{c}\text { Maximum } \\
\text { batch size }\end{array}$ \\
\hline Preparation & 80 & 5 & 1.20 & 0.12 & 10 & 200 \\
Reaction 1 & 100 & 8 & 18.34 & 0.01 & 8 & 180 \\
Reaction 2 & 150 & 6 & 11.73 & 0.01 & 5 & 150 \\
Reaction 3 & 100 & 8 & 14.50 & 0.01 & 8 & 180 \\
Packing 1 & 50 & 2 & 1.00 & 0.14 & 10 & 190 \\
Packing 2 & 50 & 2 & 1.00 & 0.14 & 10 & 190 \\
Drumming 1 & 20 & 5 & 0.80 & 0.12 & 5 & 150 \\
Drumming 2 & 20 & 5 & 0.80 & 0.12 & 5 & 150 \\
\hline
\end{tabular}


Table S3. Covariance of production demands

\begin{tabular}{ccccc}
\hline & P1 & P2 & P3 & P4 \\
\hline P1 & 10 & 2.5 & 2.8 & 0 \\
P2 & 2.5 & 10 & 0 & 1.9 \\
P3 & 2.8 & 0 & 10 & 0 \\
P4 & 0 & 1.9 & 0 & 10 \\
\hline
\end{tabular}

\section{Appendix C: Dual formulations for second-stage of the ARO model for scheduling}

\section{application}

Although the corresponding dual formula is given in Shi's work ${ }^{9}$, there are some errors in their formula.

Besides, the derived dual formulas are also different because different optimization schemes are adopted. The dual formulations are shown as follows:

$$
\begin{aligned}
& \min _{\text {dual variables }} \text { dualobj }=-\sum_{i} \sum_{n}\left(f d_{i} \cdot d w s_{i n} \cdot D A_{i n}\right)+\sum_{i} \sum_{n}\left[\text { htot } \cdot\left(1-d w s_{i n}\right) \cdot D B_{i n}\right] \\
& +\sum_{i} \sum_{n}\left[\text { htot } \cdot\left(1-d w s_{i n}\right) \cdot D C_{i n}\right]+\sum_{i} \sum_{n>1}\left(\text { htot } \cdot d w s_{i n} \cdot D D_{i n}\right)+\sum_{i} \sum_{n>1}\left[\text { htot } \cdot\left(1-d w f_{\text {in }}\right) \cdot D F_{\text {in }}\right] \\
& +\operatorname{htot} \cdot \sum_{n} D I_{n}+\sum_{i} \sum_{n}\left(\text { htot } \cdot D J_{i n}\right)-\sum_{i} \sum_{n}\left(b_{i}^{\min } \cdot d w s_{i n} \cdot D K_{i n}\right)+\sum_{i} \sum_{n}\left(b_{i}^{\max } \cdot d w s_{i n} \cdot D L_{i n}\right) \\
& -\sum_{i} \sum_{n}\left(b_{i}^{\min } \cdot d w f_{i n} \cdot D M_{i n}\right)+\sum_{i} \sum_{n}\left(b_{i}^{\max } \cdot d w f_{i n} \cdot D N_{i n}\right) \\
& -\sum_{i} \sum_{n}\left[b_{i}^{\min }\left(\sum_{n^{\prime}<n} d w s_{i n^{\prime}}-\sum_{n^{\prime} \leq n} d w f_{i n^{\prime}}\right) \cdot D P_{i n}\right]+\sum_{i} \sum_{n}\left[b_{i}^{\max }\left(\sum_{n^{\prime}<n} d w s_{i n^{\prime}}-\sum_{n^{\prime} \leq n} d w f_{i n^{\prime}}\right) \cdot D Q_{i n}\right] \\
& +\sum_{i} \sum_{s} \sum_{n}\left(s i_{s i} \cdot b_{i}^{\max } \cdot m_{i s} \cdot d w s_{i n} \cdot D U_{i s n}\right)+\sum_{i} \sum_{s} \sum_{n}\left(s o_{s i} \cdot b_{i}^{\max } \cdot m_{i s} \cdot d w f_{i n} \cdot D V_{i s n}\right) \\
& +\sum_{s}\left(\operatorname{sint}_{s} \cdot D 3_{s}\right)+\sum_{s} \sum_{n}\left(c_{s} \cdot D 5_{s n}\right)-\sum_{k} \sum_{s p}\left(\mathbf{s}_{k, s p} \cdot \mathbf{g}_{k, s p}\right)
\end{aligned}
$$

$$
\begin{array}{ll}
\text { s.t. } & -D M_{i n}+D N_{i n}-\sum_{s}\left(s o_{s i} \cdot m_{i s} \cdot D T_{i s n}\right)+D X_{i} \geq 0, \quad \forall i, n=1 \\
& -D M_{i n}+D N_{i n}-D R_{i n}-\sum_{s}\left(s o_{s i} \cdot m_{i s} \cdot D T_{i s n}\right) \geq 0, \quad \forall i, n>1 \\
& -D P_{i n}+D Q_{i n}+D R_{i(n+1)}+D W_{i} \geq 0, \quad \forall i, n=1 \\
& -D P_{i n}+D Q_{i n}-D R_{i n}+D R_{i(n+1)} \geq 0, \quad \forall i, 1<n<|N| \\
& -D P_{i n}+D Q_{i n}-D R_{i n}+D 8_{i} \geq 0, \quad \forall i, n=|N|
\end{array}
$$




$$
\begin{aligned}
& v d_{i} \cdot D A_{i n}-D K_{i n}+D L_{i n}+D R_{i(n+1)}-\sum_{s}\left(s i_{s i} \cdot m_{i s} \cdot D S_{i s n}\right) \geq-v c_{i}, \quad \forall i, n<|N| \\
& v d_{i} \cdot D A_{i n}-D K_{i n}+D L_{i n}-\sum_{s}\left(s i_{s i} \cdot m_{i s} \cdot D S_{i s n}\right)+D Z_{\text {in }} \geq-v c_{i}, \forall i, n=|N| \\
& s i_{s i} \cdot D S_{i s n}+s i_{s i} \cdot D U_{i s n}+s i_{s i} \cdot D 3_{s} \geq 0, \quad \forall i, s, n=1 \\
& s i_{s i} \cdot D S_{i s n}+s i_{s i} \cdot D U_{i s n}+s i_{s i} \cdot D 4_{s n} \geq 0, \quad \forall i, s, 1<n<|n| \\
& s i_{s i} \cdot D S_{i s n}+s i_{s i} \cdot D U_{i s n}+D 2_{i s}+s i_{s i} \cdot D 4_{s n} \geq 0, \quad \forall i, s, n=|n| \\
& \text { so }_{s i} \cdot D T_{i s n}+s o_{s i} \cdot D V_{i s n}+D Y_{i s} \geq 0, \quad \forall i, s, n=1 \\
& \text { so }_{s i} \cdot D T_{i s n}+s O_{s i} \cdot D V_{i s n}-s o_{s i} \cdot D 4_{s n} \geq 0, \quad \forall i, s, n>1 \\
& -D A_{i n}+D B_{i n}-D C_{i n}+D E_{i n} \geq 0, \quad \forall i, n \\
& -\operatorname{snp}_{s} \cdot D 6_{s}-\operatorname{snp} p_{s} \cdot D 7_{s} \geq s n p_{s} \cdot p_{s}, \quad \forall s \\
& D 3_{s}-D 4_{s(n+1)}+D 5_{s n} \geq 0, \quad \forall s, n=1 \\
& D 4_{s n}-D 4_{s(n+1)}+D 5_{s n} \geq 0, \quad \forall s, 1<n<|N| \\
& D 4_{s n}+D 5_{s n}+s n p_{s} \cdot D 6_{s} \geq 0, \quad \forall s, n=|N| \\
& -\sum_{i} D G_{i n}+D G H+D H_{(n+1)}+D I_{n} \geq 0, \quad n=1 \\
& -\sum_{i} D F_{i n}-\sum_{i} D G_{i n}-D H_{n}+D H_{(n+1)}+D I_{n} \geq 0, \forall 1<n<|N| \\
& -\sum_{i} D F_{i n}-\sum_{i} D G_{i n}-D H_{n}+D I_{n} \geq 0, \quad n=|n| \\
& -D B_{i n}+D C_{i n}-D D_{i(n+1)}+D E_{i(n+1)}+D F_{i(n+1)}+D J_{i n} \geq 0, \quad \forall i, n=1 \\
& -D B_{i n}+D C_{i n}+D D_{i n}-D D_{i(n+1)}-D E_{i n}+D E_{i(n+1)}+D F_{i(n+1)}+D J_{i n} \geq 0, \quad \forall i, 1<n<|N|(C \\
& -D B_{i n}+D C_{i n}+D D_{i n}-D E_{i n}+D J_{i n} \geq 0, \quad \forall i, n=|N| \\
& D B_{i n}-D C_{i n}+D G_{i n} \geq 0, \quad \forall i, n \\
& \underline{d 7_{s p}=D 7_{s}, \quad|s|-3 \leq s \leq|s|, 1 \leq s p \leq 4} \\
& 0 \leq \mathbf{g}_{k, s p} \leq d 7_{s p}, \quad \forall k, s p \\
& \underline{\mathbf{g}_{k, s p} \leq M_{0} v_{k}, \quad \forall k, s p}
\end{aligned}
$$




$$
\begin{gathered}
\frac{\mathbf{g}_{k, s p} \geq d 7_{s p}-M_{0}\left(1-v_{k}\right), \forall k, s p}{\sum_{k} v_{k}=1, v_{k}=\{0,1\}} \\
\left\{\begin{array}{c}
D A_{i n}, D B_{i n}, D C_{i n}, D D_{i n}, D E_{i n}, D F_{i n}, \\
D H_{n}, D I_{i n}, D J_{i n}, D K_{i n}, D L_{i n}, D M_{i n}, D N_{i n}, \geq 0 \\
D P_{i n}, D Q_{i n}, D U_{i s n}, D V_{i n}, D 5_{s n}, D 7_{s}, d 7_{s p}
\end{array}\right. \\
\left\{\begin{array}{c}
D G_{i n}, D G H, D R_{i n}, D S_{i s n}, D T_{i s n}, D W_{i n}, \\
D X_{i n}, D Y_{i n}, D Z_{i}, D 8_{i}, D 2_{i s}, D 3_{s}, D 4_{s n}, D 6_{s}
\end{array}\right. \text { Free variable }
\end{gathered}
$$

where $D A_{i n}, D B_{i n}, D C_{i n}, D D_{i n}, D E_{i n}, D F_{i n}, D G_{i n}, D G H, D H_{n}, D I_{i n}, D J_{i n}, D K_{i n}, D L_{i n}$, $D M_{i n}, D N_{i n}, D P_{i n}, D R_{i n}, D S_{i s n}, D T_{i s n}, D U_{i s n}, D V_{i n}, D W_{i n}, D X_{i n}, D Y_{i n}, D Z_{i}, D 2_{i s}, D 3_{s}$, $D 4_{s n}, \quad D 5_{s n}, D 6_{s}, D 7_{s}, d 7_{s p}, D 8_{i}, \quad \mathbf{g}, \quad v$ are the dual variables. $M_{0}$ is set as $10^{8}$ in this application, and $\mathbf{s}$ represents the extreme points of the uncertainty set. The formulations with underline are relevant with the uncertainty. 